\title{
Dinâmica de custos de uma instituição de ensino: modelo, cálculo da inflação interna e simulações
}

Ronaldo Lamounier Locatelli

$\mathrm{PhD}$ em Economia pela Universidade de Londres

Professor de Economia e Finanças do Curso de Mestrado Profissional em Administração da Fundação Pedro Leopoldo

ronaldo.locatelli@yahoo.com.br

Rubens Barbosa dos Santos

Mestre em Administração pela FPL. Analista do Senac-BH

consultoriarubens@htomail.com

Wanderley Ramalho

Doutor em Administração pelo CEPEAD/UFMG.

Professor do Curso de Mestrado Profissional em Administração da Fundação Pedro Leopoldo - FPL wanderley.ramalho@yahoo.com.br

Gustavo Rodrigues Cunha

Doutor em Business Administration pela Università di Bologna.

Professor do Curso de Mestrado em Administração do Centro Universitário UniHorizontes.

Gustavo.cunha@unihorizontes.br

Editor Científico: José Edson Lara

Organização Comitê Científico

Double Blind Review pelo SEER/OJS

Recebido em 05.04.2019

Aprovado em 08.11.2019

Este trabalho foi licenciado com uma Licença Creative Commons - Atribuição - Não Comercial 3.0 Brasil 
Objetivo do estudo: Desenvolver uma metodologia de análise da dinâmica dos custos e estimar a inflação interna da instituição.

Metodologia / abordagem: O modelo de formação de preços foi construído baseado na abordagem de mark up. Sua operacionalização demandou o uso do instrumental de números índices. Para ilustrar sua pertinência, a metodologia foi aplicada a uma unidade do Ser viço Nacional de Aprendizagem Comercial (Senac).

Originalidade / Relevância: $\mathrm{O}$ artigo contém inovações e apresenta de forma consistente um modelo que permite acompanhar os custos e mensurar a inflação interna de uma empresa. $\mathrm{O}$ modelo desenvolvido pode, também, ser usado para projetar os custos da instituição, tendo em vista as previsões macroeconômicas.

Principais resultados: $\mathrm{O}$ modelo se mostrou apropriado para apurar os custos e acompanhar a inflação interna da instituição, permitindo reproduzir a trajetória observada nos custos da unidade nos anos recentes.

Contribuições teóricas / metodológicas: Afigura-se viável o uso da metodologia em diferentes organizações produtivas, bastando, para isso, selecionar indicadores que reflitam os custos internos de forma análoga à apresentada no artigo.

Contribuições sociais / para a gestão: $O$ desenvolvimento metodológico e as aplicações apresentadas neste artigo são de utilidade não apenas para o Senac, mas também para os gestores de diferentes organizações, uma vez que uma boa gestão dos custos e precificação adequada de produtos e serviços constituem tarefas imprescindíveis para manter a competitividade do negócio.

Palavras-chave: Formação de Preços, Inflação Interna, Gestão de Custos, Mark Up

\title{
Cost dynamics of an educational institution: model, internal inflation calculation and simulations
}

\begin{abstract}
Study Objective: To develop a methodology for cost dynamics analysis and to estimate the institution's internal inflation.

Methodology / Approach: The pricing model was built based on the mark up approach. Its operationalization required the use of the instrument of index numbers. To illustrate its relevance, the methodology was applied to a unit of the National Service for Commercial Learning (Senac).

Originality / Relevance: The article contains innovations and consistently presents a model for tracking costs and measuring a company's internal inflation. The developed model can also be used to project the institution's costs, taking into account the macroeconomic forecasts.
\end{abstract}


Main Results: The model proved to be appropriate to calculate costs and to follow the internal inflation of the institution, allowing to reproduce trajector observed in unit costs in recent years.

Theoretical / Methodological Contributions: It is feasible to use the methodology in different productive organizations, simply selecting indicators that reflect internal costs in a similar way to that presented in the article.

Social contributions / to management: The methodological development and applications presented in this article are of use not only to Senac, but also to managers of different organizations, considering that good cost management and proper pricing of products and services constitute essential tasks to maintain the competitiveness of the business.

Keywords: Price Formation, Internal Inflation, Costs Management, Mark Up.

\section{Dinámica de costos de una institución educacional: modelo, cálculo de la inflación interna y simulaciones}

\section{Resumen}

Objetivo del estudio: Objetivo del estudio: Desarrollar una metodología para analizar la dinámica de costes y estimar la inflación interna de la institución.

Metodología/enfoque: El modelo de formación de precios se creó sobre la base del enfoque de mark up y requerió el uso de índices de precios. Para ilustrar su pertinencia, la metodología se aplicó a una unidad del Servicio Nacional de Aprendizaje Comercial (Senac).

Originalidad / Relevancia: El artículo contiene innovaciones y presenta un modelo que le permite realizar un seguimiento de los costes y medir la inflación interna de una empresa. El modelo desarrollado también puede utilizarse para proyectar los costos de la institución, en vista de las previsiones macroeconómicas

Principales resultados: El modelo resultó apropiado para determinar los costos y monitorear la inflación interna de la institución, permitiendo reproducir la trayectoria observada en los costos de la unidad en los últimos años.

Contribuciones teóricas/metodológicas: La metodología puede ser utilizada en distintas organizaciones, siempre que se tengan en cuenta las especificidades de las respectivas estructuras de costo.

Contribuciones sociales / a la gestión: El desarrollo metodológico y las aplicaciones presentadas en este artículo son de utilidad no sólo para Senac, sino también para los gerentes de diferentes organizaciones, ya que una buena gestión de costos y precios productos y servicios son tareas esenciales para mantener la competitividad del negocio.

Palabras clave: Formación de Precios; Inflación Interna; Gestión de Costes, Mark Up 


\section{Introdução}

O ambiente de negócios no qual os agentes econômicos ofertam produtos e serviços e realizam suas transações tem se tornado cada vez mais competitivo. Grande parte dessa dinâmica advém, direta ou indiretamente, do fenômeno da globalização, caracterizado pela concorrência em escala global, do alargamento das fronteiras de atuação das empresas e do uso intenso das tecnologias de informação e comunicação (Kotler, 2011). Em decorrência desse ambiente em constante mutação, organizações bem-sucedidas têm promovido mudanças em suas estruturas organizacionais e em seus processos de negócios, tornando-se empresas com foco em resultados e utilização de estruturas mais flexíveis e/ou a atuação em redes de empresa (Rogers, 2017; Pant e Yu, 2018).

$\mathrm{O}$ aumento da competitividade entre as empresas demanda maiores esforços dos gestores no desenvolvimento de ações que facilitem o alcance dos objetivos voltados para a manutenção e melhoria de posições sustentáveis no mercado, por meio de estratégias que estabeleçam diferenciais competitivos (Lechner e Kreutzer, 2010; Farjoun, 2002). De acordo com Porter (2008), a vantagem competitiva pode advir da capacidade de produzir com custos mais baixos ou de se diferenciar, destacando a empresa aos olhos dos clientes e, com isso, conseguindo operar com preços mais elevados. Nessas circunstâncias, a gestão de custos ganha particular interesse não somente por ser a base que sustenta a diferenciação, como também por afetar a lucratividade e a sobrevivência das empresas (Sigalas e Papadakis, 2018).

Estratégias de negociação com fornecedores, controle e gerenciamento dos custos internos podem ser fatores decisivos em um cenário de acirramento da competitividade. Repassar os custos aos preços não é tarefa fácil e requer controle e conhecimento profundo das etapas de produção e comercialização dos produtos da empresa, demandando instrumento apropriado que permita o acompanhamento e o monitoramento das despesas. Esse instrumento pode ser utilizado tanto para análises gerenciais de curto prazo, quanto para o planejamento e formulação de estratégias de longo prazo. Precificar produtos e serviços assume, assim, um papel importante na gestão empresarial, especialmente em um país como o Brasil que tem convivido com altas taxas inflacionárias (Abreu, 2014; Franco, 1995; Lopes, 1986). Embora tenha sido observado o fim do regime de superinflação vivenciado pelo país de 1980 a junho de 1994, como resultado do bem-sucedido Plano Real 
de Estabilização, a taxa de elevação dos preços ainda é alta em comparação com às das economias avançadas, de tal forma que as empresas não podem se descuidar da evolução de seus custos vis-à-vis a de seus concorrentes.

Existem vários índices que retratam a evolução dos preços em setores e regiões do país, porém nenhum deles é desenvolvido de modo específico para retratar a evolução dos custos de uma organização particular. Daí advém a necessidade de se mensurar a variação dos custos no âmbito das empresas, para evitar que seus preços fiquem defasados ou estabelecidos de modo inconsistente com a lógica do mercado. Essa preocupação com o nível e evolução dos preços se aplica não apenas aos setores agrícolas, industriais e comerciais, mas também à prestação de serviços. Nesse caso, merece ênfase especial o setor de educação no segmento de fundações, que contribui para a expansão do ensino e para o desenvolvimento econômico, mas enfrenta uma concorrência acirrada, em um ambiente de crise econômica e de redução abrupta de incentivos do governo com cortes do Fundo de Financiamento Estudantil (FIES) a partir de 2015 (Franca, 2017; Chaves e Amaral, 2015).

Não obstante a importância do tema são escassos estudos acadêmicos que versam sobre a elaboração de um consistente sistema de acompanhamento da inflação em empresas educacionais. Este estudo procura suprir parte desta lacuna ao desenvolver uma metodologia apropriada para analisar a dinâmica dos preços e os seus impactos na inflação de uma instituição de ensino. A metodologia permite elaborar um índice que é capaz de acompanhar a evolução dos custos internos e de possibilitar análises de cenários em face de possíveis modificações da economia nacional.

\section{0 referencial teórico}

O levantamento e o acompanhamento do índice de inflação interna consistem em importante instrumental que deveria ser utilizado para subsidiar a gestão das instituições, tendo em vista as variações de preços dos bens e serviços que ocorrem ao longo do tempo nas economias. Esta recomendação é especialmente válida para países como o Brasil, que carece de bons fundamentos macroeconômicos necessários à estabilidade dos preços (OECD, 2018).

\subsection{Custos e estratégias de precificação}


Reconhece-se o papel representado pela diferenciação dos produtos na conquista de mercado, mas obviamente não se pode descuidar do preço como uma das variáveis fundamentais para tornar economicamente viável a oferta de bens ou serviços por parte de uma empresa a seus clientes (Sharaaz, 2016). Segundo Hoji (2012), a determinação do preço de venda é uma questão fundamental para qualquer empresa, pois se ela praticar um preço muito alto inibirá a venda e, se o preço for muito baixo, poderá não gerar retorno esperado. Pires (2004) chama a atenção para as práticas não sustentáveis de preços baixos e altos, uma vez que os primeiros podem ocasionar elevação de vendas com perda na qualidade do atendimento ao cliente, enquanto os preços elevados reduzem vendas ocasionando ociosidade das máquinas, equipamentos ou das pessoas.

Dessa forma, no processo de precificação de um produto/serviço é necessário que os gestores analisem quanto vale o produto na ótica dos clientes, devendo buscar a eficiência das operações e estar atentos às modificações da economia e dos desejos e necessidades dos consumidores (Nan et al., 2018). A precificação correta deve garantir à empresa competitividade no mercado e lucros compatíveis com os riscos assumidos (Fama e Stern, 2016). Para tanto, é de suma importância que os gestores acompanhem e monitorem os custos e despesas da atividade, e dominem os conceitos subjacentes (Nagle e Holden, 2003). Neste aspecto, destacam-se o custo marginal e o mark up. O custo marginal é o custo incorrido pela produção de uma unidade adicional do produto, enquanto que o mark up corresponde ao excesso do preço de venda em relação aos custos, com o objetivo de propiciar o lucro da empresa (Baye, 2010; Martins, 2010; Bornia, 2010).

O modelo de fixação de preços de acordo com o custo marginal é a formulação clássica em regime de concorrência perfeita, e muito bem relatado por autores, como Simonsen (1993). Contudo, não obstante o seu interesse acadêmico, esse modelo de preço variável (flex price) é utilizado apenas em circunstâncias especiais (Locatelli \& Beltrão, 1991). Na prática, as empresas adotam, em geral, o modelo baseado em margem de lucro que incide sobre o custo de produção. Este modelo de preço fixo (fix price) é, também, conhecido como o de mark up (Labini, 1984; Locatelli e Beltrão, 1991), e pode ser expresso matematicamente da seguinte forma: 


$$
\mathrm{RT}_{\mathrm{t}}=\mathrm{CT}_{\mathrm{t}}+\mathrm{LT}_{\mathrm{t}}
$$

Sendo:

$\mathrm{RT}_{\mathrm{t}}=$ receita total;

$\mathrm{CTt}=$ custo total;

$\mathrm{LT}_{\mathrm{t}}=$ lucro total.

A receita total é o resultado da multiplicação dos preços (P) pela quantidade (Q) produzida/vendida:

$$
\mathrm{RT}_{\mathrm{t}}=\mathrm{P}_{\mathrm{t}} \times \mathrm{Q}_{\mathrm{t}}
$$

Fixando a margem de lucro (m) como proporção dos custos, tem-se:

$$
\mathrm{m}=\mathrm{LT}_{\mathrm{t}} / \mathrm{CT}_{\mathrm{t}}
$$

Reescrevendo a equação, obtém-se:

$$
\mathrm{LT}_{\mathrm{t}}=\mathrm{m} \times \mathrm{CT}_{\mathrm{t}}
$$

Assim, a equação (1) pode ser reescrita como:

$$
\mathrm{P}_{\mathrm{t}} \times \mathrm{Q}_{\mathrm{t}}=\mathrm{CT}_{\mathrm{t}}+\left(\mathrm{m} \times \mathrm{CT}_{\mathrm{t}}\right)
$$

Segue-se que, de acordo com a especificação deste modelo, o preço de venda é igual a força do mark up $(1+\mathrm{m})$ multiplicada pelo custo unitário de produção $($ Cunit = CT/Q).

$$
\mathrm{P}_{\mathrm{t}}=(1+\mathrm{m}) \text { Cunit }_{\mathrm{t}}
$$

Este método de cálculo do preço de venda, parte do custo apurado internamente (custo de dentro para fora), sendo acrescido ao custo do produto uma margem (mark up), definida para cobrir os demais gastos que não estão presentes no custo do produto e o lucro desejado pelos proprietários da empresa. O uso dessa abordagem demanda identificação dos custos e despesas de forma bem clara para assegurar uma precificação aderente aos fundamentos da empresa.

\subsection{Os preços dos produtos e serviços e o índice de inflação interna}

A inflação reflete um fenômeno complexo, e tem como efeito a desvalorização da moeda que provoca o desalinhamento dos preços relativos, afetando as famílias e as empresas (Sachs e Larrain, 2000; Blanchard, 1999). Assim, instrumentais e métodos adequados para a apuração de custos e acompanhamento dos preços devem ser usados para retratar a situação de setores e mesmo de empresas específicas, fornecendo informações importantes na tomada de decisões estratégicas. 
Em uma perspectiva macroeconômica e regional há no país vários índices de preços, apurados por reputadas instituições de pesquisa. Esses índices, além de revelarem a evolução dos preços, são muito utilizados como indexadores para corrigir itens de custos/despesas das empresas. Contudo, eles devem ser usados com parcimônia, pois a estrutura de ponderação utilizada nesses índices é largamente diferente da estrutura de custos de qualquer empresa produtiva. Destarte, não podem ser considerados substitutos perfeitos de um índice genuíno construído com o propósito de apurar a inflação interna de uma organização. Não obstante, esses índices constituem importante insumo na construção de índices de inflação interna, conforme detalhado a seguir.

O modelo delineado neste artigo (equação 6) revela como determinar o preço de venda (valor absoluto) a partir dos custos de produção e da margem desejada de lucro. Em termos matemáticos, a taxa necessária de reajustes dos preços decorrentes dos os efeitos da inflação nos custos da empresa, considerando o mark up constante, pode ser obtido a partir da equação (6), como se segue.

A equação 6 com um período de defasagem $(\mathrm{t}-1)$ assume a seguinte forma:

$$
\mathrm{P}_{\mathrm{t}-1}=(1+\mathrm{m}) \text { Cunit }_{\mathrm{t}-1}
$$

Sabe-se que a variação relativa de preços é dada pela expressão:

$$
\frac{P_{t}-P_{t-1}}{P_{t-1}}
$$

Assim, utilizando-se as expressões 6 e 7, obtém-se:

$$
\frac{P_{t}-P_{t-1}}{P_{t-1}}=\frac{(1+m) \text { Cunit }_{t}-(1+m) \text { Cunit }_{t-1}}{(1+m) \text { Cunit }_{t-1}}
$$

Portanto,

$$
\frac{P_{t}-P_{t-1}}{P_{t-1}}=\frac{(1+m)\left[\text { Cunit }_{t}-\text { Cunit }_{t-1}\right]}{(1+m) \text { Cunit }_{t-1}}
$$

A equação 10 mostra que o preço do produto/serviço deve ser reajustado de acordo com a variação do custo unitário observado na empresa no período considerado, corrigido pelas força do mark up $(1+\mathrm{m})$.

O modelo pode ter um uso mais restrito e ser empregado para acompanhar a elevação dos custos incorridos no processo produtivo (inflação interna), bastando para isso que seja desconsiderada qualquer modificação na política de fixação dos preços (expressa pela margem - mark up). Assim, pode ser usado por qualquer empresa, independentemente da 
estrutura de mercado no qual ela atua (monopólio, oligopólio ou concorrência oligopolística). A equação (11), que retrata a inflação interna, assume a forma simplificada:

$$
\frac{P_{t}-P_{t-1}}{P_{t-1}}=\frac{\Delta \text { Cunit }}{\text { Cunit }_{t}-\text { Cunit }_{t-1}}
$$

Evidentemente, que a adoção do modelo para subsidiar as estratégias de formação de preço, requer, além dos fatores internos, a análise das condições do mercado em que a empresa está inserida, devendo ser considerada a presença de competidores, os quais podem ser beneficiados por uma equivocada política de preços de seus pares. Em função da concorrência e na busca de maior competitividade no mercado, ajustes em seus processos internos e externos podem ser necessários, com negociações e modificações de fornecedores e, até mesmo, mudanças nas estratégias de negócios. Todavia, esses ajustes passam pelo gerenciamento dos custos, de forma que o correto cálculo da inflação interna da instituição, baseado em uma metodologia apropriada, deveria ser incorporada ao sistema gerencial de qualquer empresa.

Registra-se, também, que a construção do índice de inflação interna não tem a pretensão de analisar as causas do aumento de custos, que são bem documentadas nos livros textos de economia. De acordo com a teoria econômica, desconsiderando-se a componente de expectativas (adaptativa ou racional) a inflação é resultado de choques de demanda e/ou de oferta agregada (Mankiw, 2009; Parkin e Bade, 1985). Os choques de demanda têm origem variada, mas em geral são ocasionados por políticas monetárias e físcais expansionistas. Por sua vez, um choque de oferta relaciona-se às modificações nos preços de insumos importantes, como é o caso da energia e produtos agrícolas. Independentemente de sua origem as elevações dos preços macroeconômicos afetam as empresas devido aos repasses de custos observados no mercado (Blanchard, 1999).

Tendo em vista a história inflacionária brasileira, pavimentada pelo estrutural desequilíbrio fiscal, vários autores são defensores do cálculo da inflação interna pelas corporações (Morato, 2007; Gazzana, 2004). Certamente, o cálculo da inflação interna de forma rotineira é capaz de: aprimorar a administração de custos e despesas, com foco nos itens de maior relevância ou de crescimento acima da média; estabelecer os limites dos preços de compra nas negociações com os fornecedores; e subsidiar a revisão dos preços e a fixação de novos contratos de fornecimento (Bugelli, 1983). O índice de inflação interna 
constitui, dessa forma, um instrumento gerencial adequado para subsidiar o planejamento orçamentário e avaliar o desempenho das diversas áreas de uma organização.

Parisi (1999) destaca, também, a relevância gerencial da apuração do índice, mesmo na presença de pequenas inflações. Mas para o autor o método é pouco utilizado devido às mudanças nos fatores de produção utilizados pela empresa, ou pelo menos devido à alteração nas ponderações. De fato, alterações na representatividade dos insumos nos custos de produção constituem elemento muito importante e podem dificultar o acompanhamento dos custos efetivos de uma organização. Mas isto não deve ser motivo para afastar o uso do instrumental, pois da mesma forma que a apuração de índices de preços ao consumidor comporta revisão das ponderações (fornecidas pela estrutura de consumo das famílias) com o passar do tempo, o mesmo procedimento deve ser aplicado no levantamento do índice de inflação interna. Ademais, as modificações na representatividade das despesas em decorrência de mudanças de preços relativos podem ser tratadas, como será discutido na metodologia, de forma apropriada com o uso de números índices, de tal maneira que não constituem obstáculos para o uso do instrumental.

\section{Metodologia}

\subsection{Caracterização da pesquisa e unidade da análise}

A presente pesquisa se caracteriza por ser descritiva, utilizando uma abordagem quantitativa para o tratamento dos dados (Yin, 2014). O objeto do estudo é a Unidade CFP BH do Serviço Nacional de Aprendizagem Comercial (Senac), localizada em Belo Horizonte - MG. O Senac é uma instituição de direito privado com fins públicos, focada na educação profissional na área de bens, serviços e turismo. Foi instituída em 1946, por meio do DecretoLei 8.621, com uma missão inovadora no país: oferecer, em larga escala, educação profissional destinada à formação e à preparação de trabalhadores para o comércio. Entre as inovações promovidas destacam-se as empresas pedagógicas, como os hotéis-escola e os restaurantes-escola, e posteriormente complementado pelo ensino à distância por meio de cursos volantes e de unidades móveis ensejando a Universidade do Ar, transformada no Senac EAD, voltada para cursos livres e técnicos, de extensão universitária, graduação e pós-graduação. 
O Senac conta com 34 unidades no Estado de Minas Gerais, dentre elas a CFP BH, que é objeto da presente pesquisa. A unidade oferta programas de aprendizagem comercial, cursos livres e técnicos nas áreas de gestão informática, moda, beleza, saúde, turismo e hospitalidade e graduação tecnológica em gastronomia.

\subsection{Procedimentos metodológicos}

O tema inflação interna está diretamente relacionado ao conceito de números-índices, existindo duas principais fórmulas de apuração: índices de preços de Laspeyres e o índice de preços de Paasche (Bureau of Labor Statistics, 2017; Endo, 1996; Allen, 1975; Kirsten; 1975). Na presente pesquisa foi empregado o índice agregado ponderado, do tipo Laspeyres Modificado, com base em uma ponderação móvel, para estimar e monitorar a inflação interna da unidade de análise. Este índice é descrito pelas equações 12 e 13:

$$
\begin{aligned}
\text { ISenac }_{t} & =\sum_{i}^{n} \frac{C_{t}^{i}}{C_{t-1}^{i}} W_{t-1}^{i} \\
W_{t-1}^{i} & =\frac{\frac{c_{t-1}^{i}}{c_{t-2}^{i}} W_{t-2}^{i}}{\sum_{i=1}^{n} \frac{c_{t-1}^{i}}{c_{t-2}^{i}} W_{t-2}^{i}}
\end{aligned}
$$

Sendo:

$\mathrm{C}_{\mathrm{t}}^{\mathrm{i}} \quad=$ valor da despesa referente ao item i, no período $\mathrm{t}$;

$\mathrm{C}_{\mathrm{t}-1}^{\mathrm{i}}=$ valor da despesa referente ao item $\mathrm{i}$, no período $\mathrm{t}-1$,

$C_{\mathrm{t}-2}^{\mathrm{i}}=$ valor da despesa referente ao item i, no período t-2,

$\frac{\mathrm{C}_{\mathrm{t}}^{\mathrm{i}}}{\mathrm{C}_{\mathrm{t}-1}^{\mathrm{i}}}=$ custo relativo do item i no período,

$\mathrm{W}_{\mathrm{t}-1}^{\mathrm{i}}=$ peso do item $\mathrm{i}$ no total de despesa, no período $\mathrm{t}-1$,

ISenac $_{\mathrm{t}}=$ índice de inflação da empresa no período $\mathrm{t}$.

Ressalta-se que modelo não pressupõe mudanças no uso de insumos, admitindo-se, assim, uma função de produção com coeficientes fixos (Varian, 1994). A aplicação desse modelo requer dois conjuntos básicos de dados, obtidos da estrutura das despesas e da evolução dos preços internos de cada insumo no período considerado.

Para se apurar o ISenac foram utilizadas as despesas da unidade objeto do estudo referentes ao período 2013 a 2016, compreendendo as despesas operacionais e 
administrativas, desconsiderando-se as despesas de capital. As despesas foram, inicialmente, agrupadas em conjuntos, cujos preços mostram similaridades de comportamento. Após esse levantamento, foi obtida a estrutura de pesos $\left(\mathrm{W}_{\mathrm{i}}\right)$ que corresponde a proporção de todos os custos relativos a este item de despesa em relação ao custo total da empresa, anualmente. Para identificar a evolução dos vários itens de despesas foram adotados indexadores oriundos de preços ao consumidor nacional (IPCA/IBGE) e de preços setoriais (IGP/FGV), bem como de custos da própria instituição objeto da pesquisa. Definidos os pesos ( $\left.\mathrm{w}_{\mathrm{it}-1}\right)$, os indexadores de cada item de despesa, e as mudanças de preço relativos $\left(\mathrm{c}_{t} / \mathrm{c}_{\mathrm{t}-1}\right)$, apurou-se $\mathrm{o}$ índice de inflação interna da unidade (ISenac). O modelo foi, então, utilizado para avaliar a trajetória dos custos da unidade no passado recente e, após sua validação, foi empregado para simular possíveis efeitos das elevações de preços setoriais na economia brasileira nos custos dos vários subitens e na inflação interna da instituição.

\section{Análise dos resultados}

Nesta seção detalhou-se o cálculo da inflação interna e a análise de consistência do modelo para reproduzir a evolução dos custos da instituição analisada. Foram apresentadas, também, com o uso do modelo, as projeções da inflação interna da instituição com base nas previsões macroeconômicas para a economia brasileira.

\subsection{A evolução dos custos e seleção dos indexadores para o ISenac}

Verificou-se um padrão relativamente constante na estrutura das despesas da unidade pesquisada, o que permitiu expressar o peso de cada item no custo da unidade pela média simples do período. Como seria de se esperar, por ser uma instituição dedicada ao ensino, o componente que impacta com maior intensidade as despesas operacionais é o referente à folha de salários, que contribui em média com cerca de 50\% dos custos totais. Em seguida, sobressai-se a estrutura física com $25,13 \%$ e material de consumo que tem a participação em despesas de 13,5\% (Tabela 1).

Os itens que revelaram maior variação nos custos foram os referentes às despesas de pessoal, serviços de terceiros e custos de laboratórios com aumento médio anual de 7,95\%. Em seguida, figuraram os materiais de consumo e outras despesas com aumentos de $7,72 \%$ 
ao ano, respectivamente. Os menores aumentos de custos foram observados nos itens referentes à estrutura física $(6,66 \%)$.

Após o levantamento da evolução das várias despesas operacionais do Senac BH e dos vários índices de preços passíveis de refletirem a correção nominal dessas despesas, foi feita uma análise para a seleção dos indexadores do ISenac, que está detalhada na Tabela 1.

\section{Tabela 1}

Importância relativa das despesas e indexadores selecionados: ISenac, 2014-2016

\begin{tabular}{|c|c|c|c|c|}
\hline Despesas Operacionais & Peso & $\begin{array}{c}\text { Variação } \\
\text { Despesas } \\
\text { Anualizadas } \\
(\%)\end{array}$ & $\begin{array}{c}\text { Indexadores } \\
\text { de Despesa } \\
\text { Selecionados }\end{array}$ & $\begin{array}{c}\text { Diferença } \\
(\%)^{1}\end{array}$ \\
\hline Pessoal & & 7,95 & & N.D \\
\hline Salários docentes & 0,4458 & 7,72 & Correção dos & \\
\hline Salários administrativos & 0,0644 & 9,51 & salários & \\
\hline Material de Consumo & & 7,72 & IGP/DI & $-0,520$ \\
\hline Materiais diversos & 0,0839 & 7,72 & & \\
\hline Material eletrônico & 0,0078 & 7,72 & & \\
\hline $\begin{array}{l}\text { Software } \\
\text { Mobiliário }\end{array}$ & $\begin{array}{l}0,0151 \\
0,0101\end{array}$ & $\begin{array}{l}7,72 \\
7,72\end{array}$ & & \\
\hline Livros & 0,0124 & 7,72 & & \\
\hline Boletos & 0,0057 & 7,72 & & \\
\hline Estrutura Física & & 6,66 & IGPM & 0,413 \\
\hline Custo Sala Convenc. & 0,1633 & 6,10 & & \\
\hline Custo de Laboratório & 0,0880 & 7,95 & & \\
\hline Serviços de Terceiros & & 7,95 & IPCA & $-0,167$ \\
\hline Serviços de Limpeza & 0,0423 & 7,95 & & \\
\hline Serviços de Vigilância & 0,0528 & 7,95 & & \\
\hline Outras Despesas & & 7,72 & IGP/DI & $-0,520$ \\
\hline Energia Elétrica & 0,0016 & 7,72 & & \\
\hline Água e Esgoto & 0,0013 & 7,72 & & \\
\hline Manutenção & 0,0027 & 7,72 & & \\
\hline Impostos & & 7,68 & IPCA & 0,0836 \\
\hline IPTU & 0,0030 & 7,68 & & \\
\hline Total & 1,0000 & & & \\
\hline
\end{tabular}

Verifica-se que, em geral, as despesas dos diversos itens mostraram comportamento bem próximo aos dos indexadores selecionados (indexadores anualizados: IGP/DI = 7,16\%; IGPM $=7,10 \%$; e IPCA $=7,79 \%$ ). Em alguns itens, os indexadores levam a uma ligeira subestimação das despesas, e em outros uma superestimação, mas não há um claro padrão 
de comportamento. Dessa maneira, parece ser apropriado considerar que os indexadores selecionados apresentam um bom retrato da evolução das despesas dos vários itens utilizados no processo produtivo da organização.

\subsection{A inflação interna medida pelo ISenac}

Para apurar o ISenac foram empregadas as fórmulas descritas na metodologia, equações 12 e 13, considerando-se os pesos e os indexadores de despesas eleitos, detalhados na Tabela 1. Ao aplicar os dados nas fórmulas obteve-se um índice (ISenac) de 1,07613 (somatório do relativo ponderado). Ou seja, a inflação interna apurada foi de 7,613\% ao ano [(1,07613 - 1) x 100], no período de 2014 a 2016. Pode-se verificar que os principais fatores que explicaram a inflação interna foram as correções dos salários dos docentes (contribuição relativa na variação de $45,21 \%)$, custos referentes à estrutura física $(22,8 \%)$ e materiais diversos $(8,5 \%)$ (Tabela 2$)$.

\section{Tabela 2}

ISenac no período de 2014 a 2016: taxa média anual (\%)

\begin{tabular}{|c|c|c|c|c|c|}
\hline Despesas Operacionais & Relativo $^{1}$ & $\mathrm{Peso}^{2}$ & $\begin{array}{c}\text { Relativo } \\
\text { Ponderado }^{3}\end{array}$ & $\begin{array}{l}\text { Contribuição } \\
\text { na Variação }\end{array}$ & $\begin{array}{c}\text { Contribuição } \\
\text { Relativa na } \\
\text { Variação } \\
\end{array}$ \\
\hline \multicolumn{6}{|l|}{ Pessoal } \\
\hline Salários de Docentes & 1,0772 & 0,446 & 0,48018 & 0,03443 & 0,4523 \\
\hline Salários Setor Administrativo & 1,0951 & 0,064 & 0,07052 & 0,00609 & 0,0799 \\
\hline \multicolumn{6}{|l|}{ Material de Consumo } \\
\hline Materiais diversos & 1,0772 & 0,084 & 0,09032 & 0,00648 & 0,0852 \\
\hline Material eletrônico & 1,0772 & 0,008 & 0,00840 & 0,00062 & 0,0081 \\
\hline Software & 1,0772 & 0,015 & 0,01629 & 0,00116 & 0,0152 \\
\hline Mobiliário & 1,0772 & 0,01 & 0,01085 & 0,00077 & 0,0101 \\
\hline Livros & 1,0772 & 0,012 & 0,01330 & 0,00093 & 0,0122 \\
\hline Boletos & 1,0772 & 0,006 & 0,00616 & 0,00046 & 0,0061 \\
\hline \multicolumn{6}{|l|}{ Estrutura Física } \\
\hline Custo Sala Convencional & 1,061 & 0,163 & 0,17328 & 0,00994 & 0,1306 \\
\hline Custos de Laboratório & 1,0795 & 0,088 & 0,09502 & 0,0070 & 0,0919 \\
\hline \multicolumn{6}{|l|}{ Serviços de Terceiros } \\
\hline Serviços de Limpeza & 1,0795 & 0,042 & 0,04560 & 0,00334 & 0,0438 \\
\hline Serviços de Vigilância & 1,0795 & 0,053 & 0,05699 & 0,00421 & 0,0553 \\
\hline \multicolumn{6}{|l|}{ Outros insumos e Despesas } \\
\hline Energia Elétrica & 1,0772 & 0,002 & 0,00166 & 0,00015 & 0,0020 \\
\hline Água e Esgoto & 1,0772 & 0,001 & 0,00140 & 7,7E-05 & 0,0010 \\
\hline
\end{tabular}




\begin{tabular}{|c|c|c|c|c|c|}
\hline Manutenção & 1,0772 & 0,003 & 0,00288 & 0,00023 & 0,0030 \\
\hline \multicolumn{6}{|l|}{ Impostos } \\
\hline IPTU & 1,0768 & 0,003 & 0,00320 & 0,00023 & 0,0030 \\
\hline ISenac & & & 1,07613 & & \\
\hline Inflação In & & & 7,613 & 7,613 & 7,613 \\
\hline
\end{tabular}

Nota: ${ }^{1}$ Relativo obtido segundo a evolução dos indexadores de despesas (média anual) selecionados para cada item, discriminados na Tabela 1. ${ }^{2}$ Peso do item na despesa total, fornecido na Tabela $1 .{ }^{3}$ Relativo ponderado $=$ peso $\mathrm{x}$ indexador da despesa no período (média anual).

${ }^{4}$ Contribuição na variação $=($ relativo $-1,0) \mathrm{x}$ peso.

Fonte: Estimativas dos autores.

Ao contrapor o ISenac aos índices inflacionários brasileiros, constata-se que a inflação interna da instituição (7,613\% ao ano) situou-se um pouco abaixo do índice de inflação oficial do país no período (média anual do IPCA/IBGE $=7,79 \%$ ), mas acima dos reajustes (média anual) dos índices apurados pela Fundação Getúlio Vargas ( IGPM =7,10\% e IGP-DI $=7,16 \%)$.

Procurou-se verificar a consistência do ISenac, que emprega, como se discutiu, diferentes indexadores de preços para corrigir as despesas, em vez das próprias despesas ocorridas. A única exceção a esta regra para o cômputo do ISenac se deu na folha de salários, caso em que foram utilizadas as correções das remunerações efetivamente observadas na unidade.

No exame da consistência foram utilizadas as variações ocorridas nas despesas de cada item, sendo estas ponderadas de acordo com o peso médio computado no ISenac, e os resultados estão descritos na Tabela 3. 


\section{Tabela 3}

Variações efetivas das despesas do Senac BH: 2014 - 2016

\begin{tabular}{|c|c|c|c|}
\hline Despesas Operacionais & Peso & $\begin{array}{c}\text { Variações Efetivas } \\
\text { das Despesas }^{1}\end{array}$ & Relativo Ponderado \\
\hline \multicolumn{4}{|l|}{ Pessoal } \\
\hline Salários de Docentes & 0,44578 & 1,25813 & 0,56084 \\
\hline Salários do Administrativo & 0,06440 & 1,25008 & 0,08051 \\
\hline \multicolumn{4}{|l|}{ Material de Consumo } \\
\hline Materiais diversos & 0,08385 & 1,25008 & 0,10482 \\
\hline Material eletrônico & 0,00780 & 1,25008 & 0,00975 \\
\hline Software & 0,01513 & 1,25008 & 0,01891 \\
\hline Mobiliário & 0,01008 & 1,25008 & 0,01259 \\
\hline Livros & 0,01235 & 1,25009 & 0,01544 \\
\hline Boletos & 0,00573 & 1,25008 & 0,00716 \\
\hline \multicolumn{4}{|l|}{ Estrutura Física } \\
\hline Custo Sala Convencional & 0,16333 & 1,21345 & 0,19819 \\
\hline Custos de Laboratório & 0,08803 & 1,19432 & 0,10513 \\
\hline \multicolumn{4}{|l|}{ Serviços de Terceiros } \\
\hline Serviços de Limpeza & 0,04225 & 1,25008 & 0,05282 \\
\hline Serviços de Vigilância & 0,05280 & 1,25008 & 0,06600 \\
\hline \multicolumn{4}{|l|}{ Outros insumos e Despesas } \\
\hline Energia Elétrica & 0,00155 & 1,25008 & 0,00194 \\
\hline Água e Esgoto & 0,00130 & 1,25005 & 0,00163 \\
\hline Manutenção & 0,00268 & 1,25007 & 0,00334 \\
\hline \multicolumn{4}{|l|}{ Impostos } \\
\hline IPTU & 0,0030 & 1,2486 & 0,00371 \\
\hline Índice & 100,00 & & 1,24277 \\
\hline Índice acumulado - 2014 a 2016 & & & 24,2770 \\
\hline Variação - média anual $(\%)^{1}$ & & & 7,5138 \\
\hline
\end{tabular}

Notas: ${ }^{1}$ Índice acumulado. ${ }^{2}$ Cálculo pelo média geométrica.

Fonte: Estimativas dos autores.

Ao contrastar os resultados apurados pelo ISenac entre 2014 e 2016 (7,613\% a.a.) que emprega indexadores de preços para refletirem as despesas operacionais - e a variação efetiva da despesa do Senac BH (7,514\%), utilizando a participação de cada item no total da despesa (peso), conforme discriminado na Tabela 3, verifica-se que a diferença entre essas medidas foi de apenas 0,099 pontos percentuais.

Constata-se, assim, que o ISenac simula muito bem a trajetória das despesas operacionais da instituição e pode ser usado para monitorar os custos e para auxiliar o planejamento 
estratégico da organização, com o emprego imediato dos índices de preços correntemente divulgados pelos institutos de pesquisa.

\subsection{Projeções do ISenac consoante às expectativas para a economia brasileira}

Análises das perspectivas macroeconômicas e projeções para variáveis reais (PIB, contas externas, situação fiscal, emprego, etc.) e nominais (inflação, juros, salários e câmbio) constituem uma tarefa difícil e muito importante. Nesse esforço participam inúmeras instituições, com destaque para o FMI, o Banco Mundial, Bancos Centrais e grandes instituições financeiras globais.

No âmbito doméstico, há uma iniciativa coordenada pelo Banco Central do Brasil (Bacen) que resulta em projeções para as principais variáveis que descrevem o desempenho da economia brasileira, divulgadas semanalmente na forma de um Relatório de Mercado intitulado Focus. O Relatório é elaborado pelo Departamento de Relacionamento com Investidores e Estudos Especiais do Bacen, apresentando os resultados da pesquisa de expectativas de mercado, consoante às previsões de mais de 100 instituições (bancos, gestores de recursos, empresas do setor real, consultorias e outras) para a economia brasileira. O Relatório Focus faz parte do arcabouço do regime monetário de metas de inflação, e o seu objetivo é monitorar a evolução das expectativas de mercado para as principais variáveis macroeconômicas, de forma a gerar subsídios para o processo de formulação da política monetária, além de fornecer importantes informações para a tomada de decisão das entidades governamentais, empresas e famílias (www.bcb.gov.br/?FOCUSRELMERC).

A metodologia de inflação interna desenvolvida neste artigo (ISenac) permite, com facilidade, utilizar as estimativas produzidas no âmbito do Relatório Focus para derivar previsões de aumentos das despesas que devem ser suportados pela empresa educacional objeto da presente análise. Para projetar o ISenac é suficiente obter informações sobre possíveis trajetórias indexadores de despesas selecionados, a saber: IPCA, IGP-DI, IGPM e aumentos salarias.

Nos exercícios apresentados a seguir foram utilizadas a estrutura de peso inicial e as projeções dos indexadores que compõem o ISenac, sendo estas fornecidas pelas expectativas de mercado expressas no Relatório Focus, divulgado em 22 de junho de 2018. Nesse Relatório, no bloco referente à mediana, descortinam-se as seguintes previsões: referente a 
2018: IPCA $=4 \%$, IGP-DI = 7,18\% e IGPM =7,04\%; expectativas para 2019: $\mathrm{IPCA}=4,1 \%$, IGP-DI $=4,45 \%$ e IGPM = 4,47\%. Entretanto, não estão disponíveis no Relatório previsões sobre os aumentos salariais. Admitiu-se, assim, que o aumento salarial médio na instituição seguirá o padrão observado no período de 2014-2016, com uma correção acima da inflação oficial (IPCA) de 0,77 pontos percentuais ao ano.

\section{Tabela 4}

Projeções do ISenc para 2018 e 2019

\begin{tabular}{|c|c|c|c|c|c|c|}
\hline & Peso & $\begin{array}{l}2018 \\
\text { Índice de } \\
\text { Reajuste }^{1}\end{array}$ & $\begin{array}{c}\text { Relativo } \\
\text { Ponderado }\end{array}$ & $\begin{array}{l}\text { Novo } \\
\text { Peso }\end{array}$ & $\begin{array}{l}2019 \\
\text { Índice de } \\
\text { Reajuste }^{1}\end{array}$ & $\begin{array}{c}\text { Relativo } \\
\text { Ponderado } \\
\end{array}$ \\
\hline \multicolumn{7}{|l|}{ Despesas } \\
\hline \multicolumn{7}{|l|}{ Pessoal } \\
\hline Salários de Docentes & 0,4458 & 1,0480 & 0,4672 & 0,4420 & 1,0528 & 0,4653 \\
\hline Salários Setor Adm. & 0,0644 & 1,0480 & 0,0675 & 0,0639 & 1,0528 & 0,0672 \\
\hline \multicolumn{7}{|l|}{ Material de Consumo } \\
\hline Materiais diversos & 0,0839 & 1,0747 & 0,0901 & 0,0853 & 1,0445 & 0,0891 \\
\hline Material eletrônico & 0,0078 & 1,0747 & 0,0084 & 0,0079 & 1,0445 & 0,0083 \\
\hline Software & 0,0151 & 1,0747 & 0,0163 & 0,0154 & 1,0445 & 0,0161 \\
\hline Mobiliário & 0,0101 & 1,0747 & 0,0108 & 0,0102 & 1,0445 & 0,0107 \\
\hline Livros & 0,0124 & 1,0747 & 0,0133 & 0,0126 & 1,0445 & 0,0131 \\
\hline Boletos & 0,0057 & 1,0747 & 0,0062 & 0,0058 & 1,0445 & 0,0061 \\
\hline \multicolumn{7}{|l|}{ Estrutura Física } \\
\hline Custo Sala Convenc. & 0,1633 & 1,0718 & 0,1751 & 0,1656 & 1,0448 & 0,1730 \\
\hline Custos de Laboratório & 0,0880 & 1,0718 & 0,0943 & 0,0893 & 1,0448 & 0,0933 \\
\hline \multicolumn{7}{|l|}{ Serviços de Terceiros } \\
\hline Serviços de Limpeza & 0,0423 & 1,0400 & 0,0439 & 0,0416 & 1,0410 & 0,0433 \\
\hline Serviços de Vigilância & 0,0528 & 1,0400 & 0,0549 & 0,0520 & 1,0410 & 0,0541 \\
\hline \multicolumn{7}{|l|}{ Outros insumos e Desp. } \\
\hline Energia Elétrica & 0,0016 & 1,0747 & 0,0017 & 0,0016 & 1,0445 & 0,0016 \\
\hline Água e Esgoto & 0,0013 & 1,0747 & 0,0014 & 0,0013 & 1,0445 & 0,0014 \\
\hline Manutenção & 0,0027 & 1,0747 & 0,0029 & 0,0027 & 1,0445 & 0,0028 \\
\hline \multicolumn{7}{|l|}{ Impostos } \\
\hline IPTU & 0,0030 & 1,0400 & 0,0031 & 0,0029 & 1,0410 & 0,0030 \\
\hline Total & 1,0000 & & & 1,0000 & & \\
\hline ISenac & & & 1,0569 & & & 1,0484 \\
\hline ISenac - variação (\%) & & & 5,69 & & & 4,84 \\
\hline
\end{tabular}


A Tabela 4 contém os resultados gerados pelo ISenac, tendo sido estimada uma elevação esperada nos custos da instituição de 5,69\% para o ano de 2018 [(1,0569 - 1,0) x 100], consoante as expectativas inflacionárias para o país. A inflação interna da organização relativamente elevada para 2018 é impactada pelos itens de despesa que tendem acompanhar a variação do IGP-DI e IGPM. Estes índices segundo os especialistas devem ser bem superiores ao índice de inflação oficial (IPCA), principalmente pelas correções esperadas dos combustíveis e de produtos importados impactados pela desvalorização do câmbio, correções estas provocadas tanto por questões externas de provável elevação da taxa de juros nos Estados Unidos quanto pelo ambiente doméstico de incertezas e de pessimismo em relação ao quadro político e da gestão pública.

Projeta-se para 2019 um quadro ligeiramente melhor, com uma variação esperada de 4,84\% nos custos do Senac BH. Assim, estima-se uma desinflação de 0,85 pontos base tendo por referência o ano de 2018, explicada pelos menores aumentos dos custos em consonância com o comportamento do IGP-DI e IGPM, e a manutenção da inflação oficial em torno de $4 \%$ no ano.

\section{Considerações finais}

Esse artigo teve por objetivo apresentar o desenvolvimento de uma metodologia apropriada para analisar a dinâmica dos custos de uma instituição de ensino. Para ilustrar sua pertinência a metodologia foi aplicada à unidade Senac BH voltada para oferta de programas de aprendizagem comercial, cursos livres e técnicos nas áreas de gestão informática, moda, beleza, saúde, turismo e graduação tecnológica em gastronomia.

A operacionalização do modelo demandou o levantamento das despesas da unidade e a identificação da representatividade de cada item no custo total da instituição. Foram, também, analisadas as correspondências existentes entre as variações dos índices de preços nacionais e das despesas dos vários itens de custos da unidade. Adotando-se um modelo de preço fixo e aplicando-se o instrumental de números-índices foi elaborado o índice de inflação interna do Senac BH, que permitiu reproduzir a trajetória observada da evolução dos custos da unidade nos anos recentes e simular as implicações de possíveis modificações dos componentes de custos no índice de inflação interna. Verificou-se que o comportamento do ISenac tende ser menos volátil do que o IGP-DI e IGPM, mas com tendência de situar-se em níveis superiores ao exibido pelo IPCA/IBGE, uma vez que é impactado pelos aumentos salariais que tem sido reajustados acima da taxa oficial de inflação do país. 
Pode-se concluir que a metodologia revelou muito apropriada para se apurar os custos e acompanhar a inflação interna de uma instituição prestadora de serviços de educação. Como contribuição adicional, projeta-se ainda a viabilidade de sua utilização em outras instituições e em distintas organizações produtivas, adaptando-se as variáveis e os indicadores que refletem os custos internos.

Como se discutiu, é notório o acirramento da concorrência na comercialização de bens e serviços, e nesse contexto é fundamental que os executivos tenham acesso a informações que permitam identificar fragilidades ou pontos fortes da organização, e se for o caso desenhar alternativas para superar os eventuais problemas e gargalos. Em suma, espera-se que o artigo possa auxiliar não apenas os gestores do Senac $\mathrm{BH}$, mas também a alta gerência de empresas de diferentes setores, na construção e acompanhamento de indicadores relevantes para uma boa gestão dos custos e para a precificação de produtos e serviços, tarefas imprescindíveis para manter a competitividade do negócio.

Como limitações do artigo descortinam-se a não consideração das despesas de capital e a pequena desagregação das despesas, dificultando uma análise mais apurada da evolução dos custos da organização. Sugere-se, assim, aplicações do modelo em diferentes organizações, trabalhando com maior nível de desagregação das despesas e incorporando, na análise, o capital investido e o custo de oportunidade desse fator de produção.

\section{Referências}

Abreu, M. P. (2014). A ordem do progresso: dois séculos de política econômica no Brasil. (2 $2^{\mathrm{a}}$ edição). Rio de Janeiro: Editora Campus.

Allen, R. G. D. (1975). Index numbers in theory and practice. London: Mac.Millan.

Baye, M. R. (2010). Economia de empresa e estratégias de negócios. McGaw-Hil Bookman: Porto Alegre.

Blanchard, O. (1999). Macroeconomia: teoria e política econômica. Campus: Rio Janeiro.

Bornia, A. C. (2002). Análise gerencial de custos. Bookman: Porto Alegre.

Bugelli, S. (1983), Inflação interna: conhecendo o custo de vida real da sua empresa. Cempre, São Paulo, pp.75.

Bureau of Labor Statistics. (2017). Producer price index (PPI): guide for price adjustment. Disponível em: https://www.bls.gov/ppi/ppiescalation.htm

Chaves, V. L. J., \& Amaral, N.C. (2015). A educação superior no Brasil: os desafios 
da expansão e do financiamento e comparações com outros países. Revista Educação em Questão, 51(37), 95-120.

Endo, S. K. (1996), Números índices. São Paulo: Atual Editora Ltda.

Fama, E. F., \& Stern, J. M. (2016). A look back at modern finance: accomplishments and limitations. Journal of Applied Corporate Finance, 18(4),10-16.

Farjoun, M. (2002). Towards an organic perspective on strategy.Strategic Management Journal, 23(7), 561-594.

Franca, A.G. (2017). Educação e mercantilização: um estudo sobre a expansão do setor de ensino superior privado no Brasil a partir da década de 1990. Revista Brasileira de Ensino Superior, 3(1), 98-111.

Franco, G. H. B. (1995). O Plano Real e outros ensaios. Rio de Janeiro: Editora Francisco Alves.

Gazzana, P. A. (2004). Inflação interna da empresa, Dissertação (Mestrado em Administração), Universidade Federal do Rio Grande do Sul, Porto Alegre, RS. 122pp.

Hoji, M. (2012), Administração financeira e orçamentária. São Paulo; Atlas.

Kirsten, J. T. (1975). Metodologia da construção de índices de preços ao consumidor - custo de vida. IPE/USP, São Paulo.

Kotler, P. (2011). Re-inventing marketing to manage the environmental imperative. Journal of Marketing, 75(40), 132-135.

Labini, P. S. (1984). Ensaios sobre desenvolvimento e preço. Rio de Janeiro: Forense.

Lechner, C., \& Kreutzer, M. (2010). Strategic initiatives: past, present and future. In P. Mazzola \& F. W. Kellemanns (Eds.), Handbook of research on strategic process. Cheltenham: Edward Elgar.

Locatelli, R. L., \& Silva, J. A. B. (1991). Câmbio real e competitividade das exportações Brasileiras", Revista Brasileira de Economia,45(4), 543-64.

Lopes, F. (1986). O Choque heterodoxo. Rio de Janeiro: Editora Campus.

Mankiw, N. G. (2009), Macroeconomics. (7 $7^{\text {a }}$ Ed.). New York: Worth Publishers.

Martins, E. (2010). Contabilidade e custos. (10ª Ed.). São Paulo: Saraiva.

Morato, L. S. (2007). Inflação Interna: um estudo de caso de uma instituição de ensino superior. Dissertação (Mestrado em Administração), Faculdade Novos Horizontes, Belo Horizonte, MG, pp.133.

Nagle, T. T., \& Holden, R. K. (2003). Estratégia e tática de preços: um guia para decisões lucrativa. ( $3^{\text {a }}$. Ed.). São Paulo: Prentice Hall. 
Nan, G., Li, X., Zhang, Z., \& Li, M. (2018). Optimal pricing for new product entry under free strategy. Information Technology and Management, 19(1),1-19.

OECD. (2018), Relatórios Econômicos - Brasil, Economic survey overview. Portuguese.pdf . Disponível em: https://www.oecd.org/eco/surveys/brazil

Pant, V., \& Yu, E. (2018). Modeling simultaneous cooperation and competition among enterprises. Business \& Information Systems Engineering, 60(1), 39-54.

Parisi, C. (1999). Uma contribuição ao estudo de índice interno de preços sob a ótica da gestão econômica, Tese (Doutorado em Economia), Universidade de São Paulo. São Paulo-SP, 226pp.

Parkin, M., \& Bade, R. (1985). Modern macroeconomics. Oxford: Philip Allan.

Pires, S, R. (2004), Gestão da cadeia de suprimentos: conceitos, estratégias, práticas e casos. São Paulo: Atlas.

Porter, M. E. (2008). The five competitive forces that shape strategy. Harvard Business Review, 86, (1), 57-71.

Rogers, D. L. (2017). Transformação digital: repensando os seu negócio para a era digital. São Paulo: Autêntica Business.

Sachs, J. D., \& F. B. Larrain. (2000). Macroeconomia em uma Economia Global. São Paulo: Makron Books.

Sigalas, C., \& Papadakis, V. M., (2018). Empirical investigation of relationship patterns between competitive advantage and superior performance. Journal of Strategy and Management; 11(1), 81-111.

Sharaaz, M. R .K. (2016), Strategy and profitability: managing profits in inflation economy. Walden University. Disponível em: https://search.proquest.com/docview/1836064810?accountid=42153

Simonsen, M. H. (1993). Teoria microeconômica. Rio de Janeiro: Editora Fundação Getúlio Vargas.

Varian, H. R. (1994). Microeconomia - Princípios Básicos. Rio de Janeiro: Editora Campus.

Yin, R. K. (2014).Case study research design and methods. ( $5^{\text {th }}$ Ed.). Thousand Oaks, CA: Sage. 